\title{
Occupational exposure as a presumable cause of subcutaneous sarcoidosis in a tannery worker - case report and review of the literature
}

\author{
Małgorzata M. Michalska-Jakubus ${ }^{1}$, Katarzyna Zdeb르, Beata Rymgayłło-Jankowska², Agnieszka Korolczuk ${ }^{3}$, \\ Tomasz Żarnowski², Dorota Krasowska ${ }^{1}$ \\ ${ }^{1}$ Department of Dermatology, Venereology and Paediatric Dermatology, Medical University of Lublin, Lublin, Poland \\ ${ }^{2}$ Department of Diagnostic and Microsurgery of Glaucoma, University Hospital No. 1, Lublin, Poland
} ${ }^{3}$ Chair and Department of Clinical Pathomorphology, Medical University of Lublin, Lublin, Poland

Adv Dermatol Allergol 2018; XXXV (1): 118-121 DOI: https://doi.org/10.5114/ada.2018.73173

Sarcoidosis is a granulomatous disease with protean manifestations, most commonly involving hilar lymph nodes, pulmonary parenchyma, skin, joint/bones and eyes. The prevalence varies markedly $(1-40 / 100,000)$ and the peak incidence typically occurs in young and middle aged adults. Females are slightly more commonly affected [1-3].

Skin lesions are polymorphic and seen in about $25 \%$ of patients, in some cases being the initial or the sole sign of the disease. They may be specific with the presence of sarcoidal granulomas or non-specific (reactive). Among specific skin lesions subcutaneous nodules are distinctly rare $[4,5]$.

The cause of the disease remains unclear. Recent epidemiological surveys support the view that some exposures in occupational or environmental settings might be antigens that elicit a specific sarcoid (granulomatous) reaction, including systemic or cutaneous sarcoidosis [2, 3, 6-8].

This report describes a long-term tannery worker who developed subcutaneous masses of the periorbital area that proved to be sarcoidal infiltrations. We also review the literature on this subject to better define the clinical features of this unusual disease subset and potential environmental risk factors.

A 59-year-old Caucasian man was referred to the Dermatological Department due to subcutaneous firm, non-tender infiltration and oedema of the left buccal area and upper eyelid with significant narrowing of the left palpebral fissure that started 2 years earlier (Figure $1 \mathrm{~A})$. The overlying skin was erythematous and non-ulcerated. The patient had been working for many years at tanning hides, had proven chrome contact allergy and otherwise no significant medical history. On ophthalmic examination no pathological changes within the left eye were detected and visual acuity was normal (V.o.u. - visus oculi utriusque, visual acuity of both eyes $=1$ ). There were no eyeball motility disturbances, and intraocular pressure was normal (13 $\mathrm{mm} \mathrm{Hg}$ ). Magnetic resonance imaging (MRI) of the orbits performed on a 1.5-T scanner revealed nonspecific thickening and prominent homogeneous enhancement of the left upper eyelid, conjunctiva and anterior part of sclera. Intraocular muscles and optic nerves were not affected with homogeneous signal intensity (Figure 2 A). A lesional biopsy revealed chronic inflammation with the presence of non-caseating granulomas within the dermis and subcutaneous tissue, consistent with sarcoidosis (Figure 2 B). Special stains for fungi (periodic acid-Schiff - PAS) and mycobacteria were negative and no foreign body was identified on polariscopic examination. Laboratory tests revealed a slightly elevated erythrocyte sedimentation rate (25/h), C-reactive protein $(10.6 \mathrm{mg} / \mathrm{l})$ and slightly increased calcium serum level (1.27 mmol/l; normal range: 0.98-1.13 $\mathrm{mmol} / \mathrm{l})$. Complete blood count, blood chemistries and urinalysis were normal. Response to purified protein derivative (PPD) of tuberculin and Quantiferon TB Gold test were negative. Neither pulmonary involvement nor hilar lymphadenopathy was detected on chest radiographs and computed tomography (CT) scans. Standard bronchofibroscopy with transbronchial lung biopsy further confirmed the absence of granulomatous inflammation. On ECG and abdominal ultrasonography no pathologies were found.

Based on the clinical appearance and diagnostic tests (histopathological examination, negative PPD of tuberculin and Quantiferon TB Gold test) subcutaneous sarcoidosis of the left periorbital area was diagnosed. Treatment

Address for correspondence: Małgorzata M. Michalska-Jakubus, Department of Dermatology, Venereology and Paediatric Dermatology, Medical University of Lublin, 13 Radziwiłłowska St, 20-080 Lublin, Poland, phone: +48 693 549 654, e-mail: mjm@poczta.onet.eu Received: 4.02.2016, accepted: 10.08.2017. 

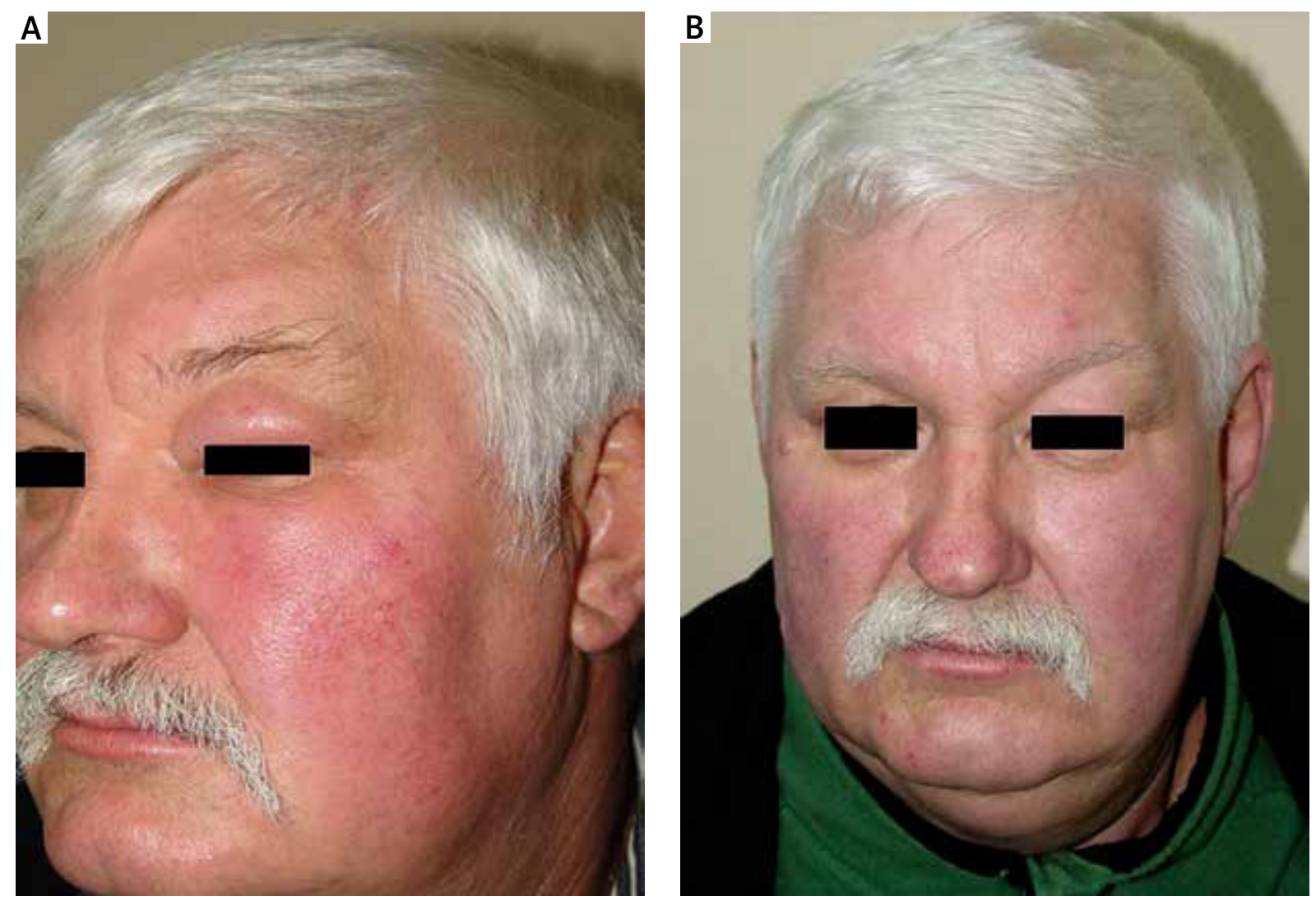

Figure 1. Subcutaneous sarcoidosis of the face in a 59-year-old Caucasian man. A - Clinical appearance at first admission: subcutaneous firm, non-tender infiltration and oedema of left buccal area and upper eyelid with significant narrowing of the left palpebral fissure; the overlying skin - erythematous, non-ulcerated. B - Clinical appearance of the patient after 6-month therapy with chloroquine $750 \mathrm{mg} /$ day: complete resolution of subcutaneous infiltration, oedema and erythema

with chloroquine $750 \mathrm{mg} /$ day was introduced, resulting in resolution of infiltration and oedema within 6 months (Figure 1 B). The patient is being regularly followed up (including lung (T) and the chloroquine is being continued in the dose of $500 \mathrm{mg} /$ day.

Subcutaneous sarcoidosis, as diagnosed in our patient, is a rare subset of the disease, first described in 1904 by Darier and Roussy, with fewer than 40 cases reported so far. It constitutes up to $12 \%$ of all cutaneous expressions of sarcoidosis. Lesions are typically multiple, asymptomatic to slightly tender, skin-coloured, firm subcutaneous nodules without overlying epidermal involvement and with a predilection for the trunk and upper extremities. However, lesions may mimic cellulitis. Histopathologically, these nodules are characterized by non-caseating naked granulomas involving fat lobules, with minimal to no septal involvement. Laboratory abnormalities are not usually noted, but if present, then typically they occur in association with abnormal radiographic findings $[4,5,9,10]$.

In the present case, subcutaneous sarcoidosis has an unusual site of occurrence in periorbital and buccal area. Isolated orbital disease is uncommon and usually involves the lacrimal gland (42.3\%), soft tissues of the orbits (38.5\%), eyelids (11.5\%) and optic nerve, and is nearly always unilateral. Soft tissues of the orbit are more frequently involved among older patients ( $>50$ years old) and women. Orbital sarcoidosis appears in 2 forms, diffuse (more commonly in patients with active systemic disease) and discrete, with a predilection for the anteroinferior quadrants of the orbit $[1,11-15]$.

Eyelid sarcoidosis appears to be much more common in women, with predilection for the lower lid. Extraocular muscle involvement is rare but has been reported in association with orbital lesions. Twenty to twenty-five percent of all patients have ophthalmic involvement. Anterior uveitis with blurred vision and photophobia is the most common manifestation, occurring in up to $85 \%$ of patients, whereas posterior uveitis is the most common cause of visual morbidity. Pathological changes may be present in the iris, cornea and conjunctiva. The most common feature at the first examination include a palpable periocular masses/infiltrations, discomfort, oedema and ptosis of the eyelid, proptosis, dry eye syndrome, double vision and decreased vision [1, 11, 12, 14, 15]. 

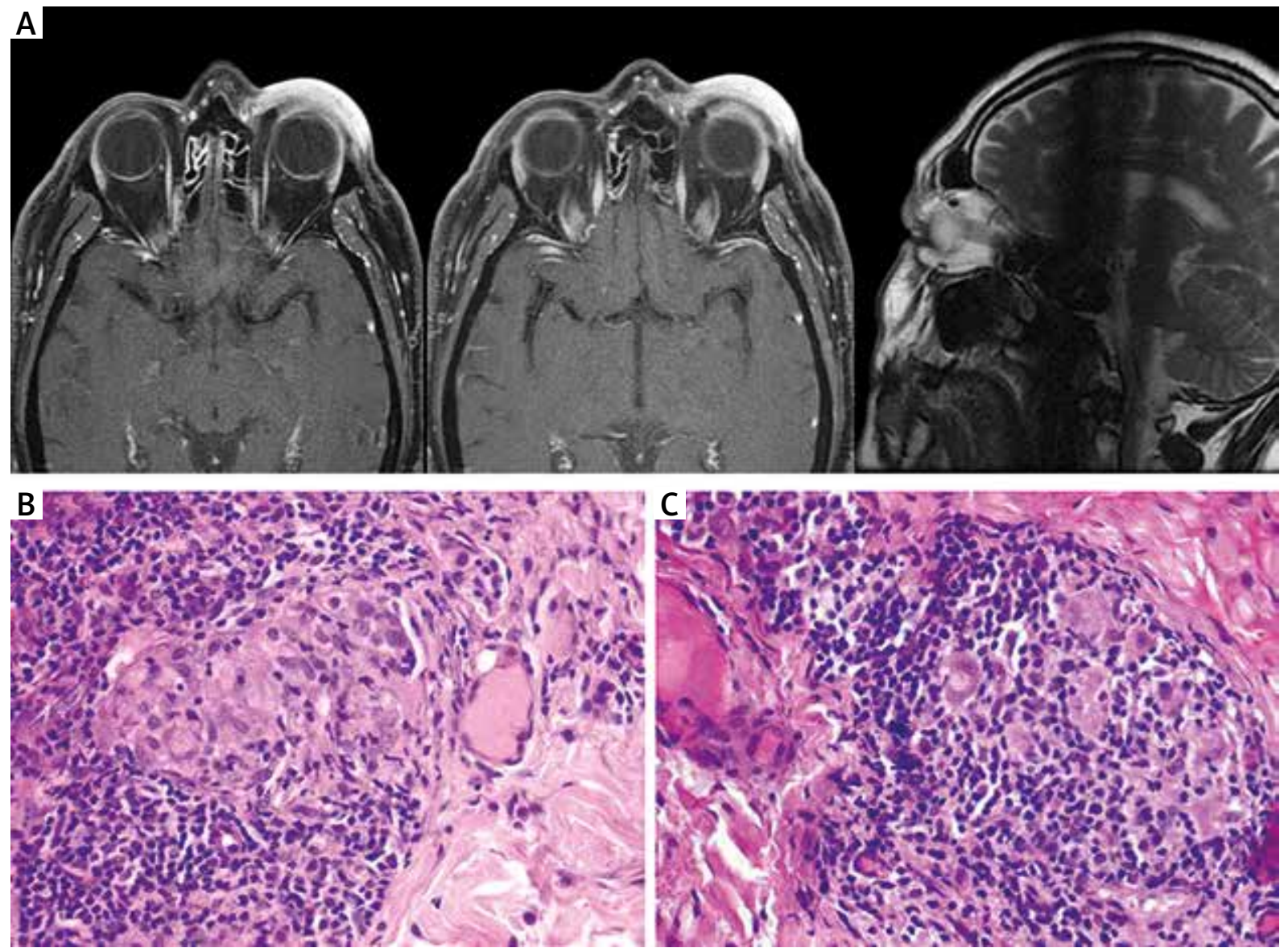

Figure 2. Diagnostic imaging. A - MRI of the orbits: nonspecific thickening and prominent homogeneous enhancement of the left upper eyelid, conjunctiva and anterior part of sclera; intraocular muscles and optic nerves not affected with homogeneous signal. B, C - Histopathology $(\mathrm{H}+\mathrm{E}$; 100x) of the lesional skin and subcutaneous tissue: chronic inflammation with the presence of non-caseating sarcoidal granulomas within the dermis and subcutaneous tissue

Several investigators have characterized the MRI findings in orbital and optic pathway sarcoidosis; however, their spectrum is unspecific. Magnetic resonance imaging may be valuable in noninvasively distinguishing orbital sarcoidosis from other neoplastic, granulomatous, and infiltrative disorders $[1,12,15,16]$. As reported by Atlas et al., the lack of hyperintensity on T2-weighted sequences may favour a diagnosis of benign infiltration, such as sarcoidosis or pseudotumour, over malignant diseases, such as lymphoma or metastasis [16].

The distinctive feature of our case is the presentation of diffuse eyelid swelling without mass lesions, even on MRI. The MRI of the orbits revealed only nonspecific thickening and prominent homogeneous enhancement of the left upper eyelid, conjunctiva and anterior part of the sclera, whereas intraocular muscles, soft tissues, the lacrimal gland and the optic nerve were unaffected. No ophthalmic involvement was revealed. A similar case of orbital sarcoidosis presenting as diffuse swelling of the lower eyelid was reported by Lee and Moon [17] in a 70 -year-old woman.
The mainstay of treatment of subcutaneous sarcoidosis is oral corticosteroids Anecdotal reports have demonstrated efficacy of oral minocycline, allopurinol, and adalimumab. The use of hydroxychloroquine has also been reported, with a response noted in 12 of 17 patients within 4 to 12 weeks in one series. For ocular disease several therapies have been proposed, including prednisone, methotrexate, azathioprine, leflunomide, and the anti-TNF agents infliximab and adalimumab; however, treatment with chloroquine, which proved to be effective in our patient, has not been reported previously [10, 18].

We hypothesize that subcutaneous sarcoidosis of the periorbital area diagnosed in our patients might be associated with working in a tannery where he was exposed to several occupational health risk factors such as benzene-based dyes and formaldehyde, aniline, butyl acetate, ethanol, benzene, toluene, sulfuric acid and ammonium hydrogen sulfide [6-8]. Another important health risk factor for tannery workers is occupational exposure to chromium ( $\mathrm{Cr}$ ), which is used as a basic tanning pigment, mainly in the organic form or in the 
protein bound-form (leather dust). Chromium may enter the body by inhalation, ingestion and by direct cutaneous contact. Professional exposure to $\mathrm{Cr}$ increases the risk of dermatitis, cutaneous ulcers, perforation of the nasal septum and respiratory illnesses [6-8]. Moreover, a review of the literature reveals that some metals, including chromium, are able to induce a local sarcoid granuloma when introduced into the skin of sensitized individuals [6-8]. Our patient had a proven chrome allergy, and skin contact with the metal might have induced sarcoid reaction, especially as the patient had the habit of rubbing the left eye area with his fingers. Although no similar cases have been described yet, two cases of fatal inhalation anthrax (one of which was complicated by systemic sarcoidosis) associated with tannery work were reported [19]. To date, several environmental risk factors associated with increased occurrence of sarcoidosis have been identified in epidemiological studies, mainly exposure to water damage, working in the metal industry, motorcycle manufacture, wood burning and machining [3]. Other exposures reported to be associated with sarcoidosis were related to air transport, hair dressing, population density, being a dental technician (berylliosis), working in the medical and health sector and manufacture of furniture [3]. Higher frequency was found in regions with metal industry and intense agriculture, especially production of potatoes, bread grains and artificial meadows. Exposure to bio-aerosols, as well as mould/mildew exposures and contact with insecticides, were reported to be associated with a higher sarcoidosis frequency by the ACCESS (A Case-Control Etiologic Study of Sarcoidosis) study [3].

Notably, ocular involvement is observed in 25-60\% of patients with systemic sarcoidosis, and it may "predate" the widespread disease. This obliges a long-term close follow-up of these patients, since they may develop systemic disease months to years later. Since eye disease may be silent, a detailed examination of the eye is strongly recommended and sarcoidosis should be considered as the cause of abnormal eye findings [1, 10-13, 15]. In our patient clinical and histopathologic features were consistent with subcutaneous sarcoidosis of the periorbital area and eyelids; however, he did not present with any extracutaneous indications in 3-year follow-up.

In conclusion, we herein present an unusual case of unilateral subcutaneous sarcoidosis of the periorbital and buccal area probably due to occupational exposure to chemical substances used in the leather tanning process.

\section{Conflict of interest}

The authors declare no conflict of interest.

\section{References}

1. Kornhauser C, Wróbel K, Wróbel K, et al. Possible adverse effect of chromium in occupational exposure of Tannery workers. Industrial Health 2002; 40: 207-13.

2. Shelley WB, Hurley HJ, Mayock RL, et al. Intradermal tests with metals and other inorganic elements in sarcoidosis and anthraco-silicosis. J Invest Dermatol 1958; 31: 301-3.

3. Rastogi SK, Pandey A, Tripathi S. Occupational health risks among the workers employed in leather tanneries at Kanpur. Indian J Occup Environ Med 2008; 12: 132-5.

4. Simon EM, Zoarski GH, Rothman MI, et al. Systemic sarcoidosis with bilateral orbital involvement: MR findings. AJNR Am J Neuroradiol 1998; 19: 336-7.

5. Dubaniewicz A. Sarkoidoza - choroba o wielu twarzach. Forum Med Rodzinnej 2009; 3: 27-41.

6. Deubelbeiss U, Gemperli A, Schindler C, et al. Prevalence of sarcoidosis in Switzerland is associated with environmental factors. Eur Respir J 2010; 35: 1088-97.

7. Błaszczyk M. Sarkoidoza skórna - różnorodność kliniczna i trudności diagnostyczne. Przegl Dermatol 2012; 99: 185-94.

8. Ahmed I, Harshad SR. Subcutaneous sarcoidosis: is it a specific subset of cutaneous sarcoidosis frequently associated with systemic disease? J Am Acad Dermatol 2006; 54: 55-60.

9. Won KY, Park SY, Lee SH. Subcutaneous sarcoidosis mimicking cellulitis. J Rheumatol 2016; 43: 674-5.

10. O’Neil JL, Moustafa F, Teague D, et al. Subcutaneous sarcoidosis without systemic involvement. Dermatol Online J 2014; 20 (8).

11. Ohara K, Judson MA, Baughman RP. Clinical aspects of ocular sarcoidosis. Eur Respir Mon 2005; 32: 188-209.

12. Prabhakaran VC, Saeed P, Esmaeli B, et al. Orbital and adnexal sarcoidosis. Arch Ophthalmol 2007; 125: 1657-62.

13. Mafee MF, Dorodi S, Pai E. Sarcoidosis of the eye, orbit, and central nervous system: role of MR Imaging. Radiol Clin North Am 1999; 37: 73-87.

14. Mavrikakis I, Rootman J. Diverse clinical presentations of orbital sarcoid. Am J Opthalmol 2007; 144: 769-75.

15. Baughman RP, Lower EE, Kaufman AH. Ocular sarcoidosis. Semin Respir Crit Care Med 2010; 31: 452-62.

16. Carmody RF, Mafee MF, Goodwin JA, et al. Orbital and optic pathway sarcoidosis: MR findings. AJNR Am J Neuroradiol 1994; 15: 775-83.

17. Lee JK, Moon NJ. Orbital sarcoidosis presenting as diffuse swelling of the lower eyelid. Korean J Ophthalmol 2013; 27: 52-4.

18. Baughman RP, Lower EE, Ingledue R, et al. Management of ocular sarcoidosis. Sarcoidosis Vasc Diffuse Lung Dis 2012; 29: 26-33.

19. Brachman PS, Pagano JS, Albrink WS. Two cases of fatal inhalation anthrax, one associated with sarcoidosis. New Engl J Med 1961; 265: 203-8. 